\title{
A Missed Opportunity for Dementia Prevention? Current Challenges for Early Detection and Modern-Day Solutions
}

\author{
R. Isaacson, N. Saif \\ Department of Neurology, Weill Cornell Medicine and NewYork-Presbyterian, New York, NY, USA
}

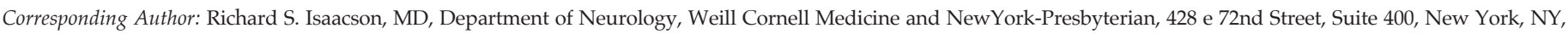
10021, USA. Email: rii9004@med.cornell.edu

The path towards developing effective therapeutics to either cure or prevent the onset of Alzheimer's disease (AD) and related neurodegenerative dementias has been plagued by challenges. Nevertheless, innovative treatments and clinical frameworks that represent our current understanding of the trajectory of disease may help to reduce morbidity, or delay symptom onset, for patients in the pre-dementia stages. Late-life AD dementia develops over an extended period, first as an asymptomatic phase referred to as preclinical $\mathrm{AD}$, which affects an estimated 46 million people in the United States alone (1). Following preclinical $\mathrm{AD}$ is the first symptomatic phase known as mild cognitive impairment (MCI) due to AD. Collectively, both stages offer a unique chance for early intervention.

However, this long window of opportunity is all too often missed by clinicians and patients at-risk. In fact, nearly half of clinical diagnoses are made in the moderate-to-severe stages of dementia. By then, the underlying disease has progressed beyond what is considered the optimal therapeutic window to mitigate cognitive decline (2). In a series of three reports, The Global Advisory Group on Future MCI Care Pathways (a working group composed of international AD and MCI experts) highlight the unmet need for an effective, broad-scale method to screen for and diagnose MCI. This working group provides an eloquent and comprehensive overview of the challenges present in the early detection of $\mathrm{MCI}$, common reasons for misdiagnosis, and present a framework of recommendations to consider for developing innovative diagnostic tools that incorporate emerging technologies.

Sabbagh et al. effectively discuss the ubiquitous benefit that can come from the implementation of widespread screening and detection of MCI for patients, clinical research, and ultimately, global public health outcomes. Not only may patients feel more motivated to explore therapeutic options and initiate evidence-based individualized lifestyle changes in collaboration with their healthcare providers, but timely $\mathrm{MCI}$ diagnoses may increase recruitment success in AD clinical trials $(3,4)$. Because of the difficulty in identifying the early stages of $\mathrm{AD}$, trial participants with accurately diagnosed Received March 10, 2020
AD may be at such an advanced stage that the benefits of treatment are limited (5). Thus, effective and reliable detection of MCI and its subtypes would allow for clinical researchers and pharmaceutical companies to evaluate therapeutics in patients who have greater margins of potential benefit.

Why aren't patients with MCI or dementia diagnosed earlier? Sabbagh et al. appropriately make the case that primary care physicians (PCPs) currently lack the assessment tools, training, time, and infrastructure necessary to efficiently detect and manage AD along its clinical continuum. Today, PCPs often refer patients with cognitive complaints to neurologists, geriatricians, neuropsychologists, and other related specialists. This approach, however, is not practical for broad-scale cognitive screening, as specialists will be unable to meet the demand of an aging population. Additionally, not all cases of $\mathrm{MCI}$ are due to $\mathrm{AD}$ or related neurodegenerative dementias, but rather secondary disorders (e.g., related to metabolism, endocrine disorders, or sleep disturbances), most of which PCPs are better equipped to evaluate for and treat.

While it is encouraging to find organizations working to train physicians on cognitive assessment and diagnosis (e.g., Clinical Partners Program), these programs are underutilized because providers are not properly incentivized (6). Clinicians have befittingly demonstrated a lack of confidence in available diagnostic tools, believing they are unreliable and put their patients at risk of misdiagnoses. Additionally, even considering the growing body of evidence suggesting the effectiveness of dementia risk reduction, there is the lingering perception that symptomatic patients can do little to delay their cognitive decline-as such, clinicians who are unclear on the potential benefits may find "no reason" to assess cognition. Recent efforts have been made to educate physicians, such as free evidence-based accredited online courses for clinicians to learn about AD risk reduction in clinical practice (AlzU.org) (7). Nevertheless, larger-scale efforts are needed to inform clinicians about the potential benefits of early cognitive assessment and risk reduction care.

Another important factor is time. PCPs often do not 
have enough time to administer cognitive assessments during routine visits. Additionally, most of the current tests need to be manually entered into the electronic medical record (EMR) system, which adds to provider workload. To mitigate this, Sabbagh et al. recommend that current practices include a trained nurse or technician for administering, scoring (if needed), and integrating the cognitive assessments into EMRs.

Although hiring and training a nurse or technician can be a significant investment of resources, the lower clerical burden may allow providers to spend more time with each patient (and/or see more patients), mitigating the financial cost. Unfortunately, insurance payers offer inadequate reimbursement for cognitive assessments, substantially decreasing a physician's incentive and ability to adopt screening procedures. Changes in government policy should be made to address this pressing issue, because without reliable reimbursement, there will continue to be a lack of cognitive assessments in primary care settings. Healthcare policy makers must understand the financial implications of early detection of $\mathrm{MCI}$, as earlier intervention can decrease financial costs of dementia care for families and the government (8).

As digital technology continues to advance, online testing has emerged as a potentially viable method for detecting subtle changes in cognition associated with preclinical AD (9). Many of these batteries are compatible across several digital platforms (e.g., smartphones, tablets, laptops), offering greater accessibility. Additionally, online testing is commonly equipped with auto-generated score reports, which lessen the administrative burden. However, even if administered online, these batteries often require direct supervision, either by a technician or healthcare provider, to verify that assessments were correctly completed and provide accurate results.

Sabbagh et al. justifiably suggest that at-home digital assessments may be the most logistical approach for broad-scale cognitive screening. If a patient is experiencing subjective cognitive decline and brings this to their physician's attention, the physician can recommend they undergo an at-home cognitive assessment before their visit. These results, which Sabbagh et al. suggest would ideally be automatically integrated into EMRs, can then be easily accessed by the physician upon seeing the patient - this time saved from an at-home cognitive assessment can then be dedicated towards collectively discussing the next steps for further evaluation and / or treatment.

However, there are important factors to consider when developing at-home digital assessments, as any variables unaccounted for can affect one's performance and thus result in an incorrectly characterized cognitive status. While the issue of technological fluency should become less relevant for future generations, older populations may not have the adequate experience with smartphones, tablets, and other digital platforms.
Similarly, patients who are already experiencing cognitive decline may find it challenging to navigate a complex digital interface. Therefore, it is important for developers to verify that future assessments require as little technological savviness as possible. Assessments must also be developed for use in heterogeneous populations. Validation in a predominantly educated, English-speaking, and/or Caucasian cohort will result in inaccurate measurements in more diverse patient groups. Accordingly, it is critical that future tests are robustly validated across various baseline characteristics, including but not limited to native language, race, ethnicity, cultural differences, sex, and education.

Along with at-home cognitive assessments, wearable biosensor technology has surfaced as an intriguing strategy to monitor cognitive function in patients. These devices, such as "fitness trackers" and smartwatches, incorporate actigraphy to continuously measure biometrics related to motor function, sleep patterns, and autonomic function. Given the body of evidence suggesting a relationship between these biometrics and $\mathrm{AD}$ pathology, clinicians may find using biosensors as a cost-efficient, non-invasive strategy to monitor their patients. A recent study in the Journal of Prevention of Alzheimer's Disease found that biosensor-collected sleep metrics correlated with performance on neuropsychological testing in patients at risk for $\mathrm{AD}$ dementia, suggesting initial feasibility of these devices for monitoring cognition (10). While further research is needed to build upon these initial results, passive technologies have become a promising strategy to overcome some of the barriers to widespread cognitive evaluation.

With each passing day, new research suggests that patients and their physicians should be proactive, rather than reactive, and take evidence-based steps to address cognitive decline, particularly in its earlier stages. To resolve the unfortunate absence of effective, universally accessible tools for cognitive assessment, collaborative efforts are needed to enact reform across a myriad of healthcare institutions, government policy, payer reimbursement, and medical education/training. Equally important, international public funding agencies (e.g., National Institutes of Health) and private foundations must prioritize adequately funding the creation of digital diagnostic and therapeutic tools that are needed for this nascent field. It is very encouraging to see a group of international experts work together to thoroughly discuss the deficiencies within the current healthcare landscape and offer a roadmap to surmount these obstacles. Now, it is critical for all stakeholders to step up and answer this urgent call to action.

Conflict of interests: Richard S. Isaacson declares that he has no conflict of interest. Nabeel Saif declares that he has no conflict of interest.

Open Access: This article is distributed under the terms of the Creative Commons Attribution 4.0 International License (http://creativecommons.org/ licenses/by/4.0/), which permits use, duplication, adaptation, distribution and 
reproduction in any medium or format, as long as you give appropriate credit to the original author(s) and the source, provide a link to the Creative Commons license and indicate if changes were made.

\section{References}

1. Brookmeyer, R., et al., Forecasting the prevalence of preclinical and clinical Alzheimer's disease in the United States. Alzheimers Dement, 2018. 14(2): p. 121-129.

2. 2019 Alzheimer's disease facts and figures. Alzheimer's \& Dementia 2019;15(3):321-87 doi: https:/ / doi.org/10.1016/j.jalz.2019.01.010[published Online First: Epub Date] I.

3. Isaacson, R.S., et al., Individualized clinical management of patients at risk for Alzheimer's dementia. Alzheimer's \& Dementia: The Journal of the Alzheimer's Association.

4. Isaacson, R.S., et al., The clinical practice of risk reduction for Alzheimer's disease: A precision medicine approach. Alzheimers Dement, 2018. 14(12): p. 1663-1673.
5. Jobke, B., et al., Setbacks in Alzheimer research demand new strategies, not surrender. PLoS Med, 2018. 15(2): p. e1002518.

6. Galvin, J.E., T.M. Meuser, and J.C. Morris, Improving physician awareness of Alzheimer disease and enhancing recruitment: the Clinician Partners Program. Alzheimer disease and associated disorders, 2012. 26(1): p. 61-67.

7. Isaacson, R.S., et al., Alzheimer's Prevention Education: If We Build It, Will They Come? www.AlzU.org. J Prev Alzheimers Dis, 2014. 1(2): p. 91-98.

8. Livingston, G., et al., Dementia prevention, intervention, and care. Lancet, 2017. 390(10113): p. 2673-2734.

9. Hackett, K., et al., Utility of the NIH Toolbox for assessment of prodromal Alzheimer's disease and dementia. Alzheimers Dement (Amst), 2018. 10: p. 764-772.

10. Saif, N., et al., Feasibility of Using a Wearable Biosensor Device in Patients at Risk for Alzheimer's Disease Dementia. J Prev Alz Dis 2020;2(7):104-111 